\title{
4 3D COCULTURE PLATFORM REVEALS INSIGHTS INTO \\ PATIENT AUTOLOGOUS IMMUNE CELL-TUMOR INTERACTION AND IMMUNE MODULATION IN VITRO
}

Garima Kaushik*, Amy Wesa. Champions Oncology, Rockville, MD, USA

Background Understanding tumor microenvironment (TME) and immune microenvironment are vital to devising therapeutic interventions against tumors. Robust models that mimic patient-specific immune interactions in vitro become necessary with the recent promise of immunotherapeutics. Organoid models recapitulate patient tumor morphology and 3D architecture but fall short in recapitulating complex immune biology of native human tumors. Infiltrating immune populations, especially tumor-infiltrating lymphocytes (TIL), vastly impact patients' response to therapies. Here we describe a co-culture platform of patient-derived xenograft (PDX) derived organoids (PDXO) with autologous TIL to simulate the tumor-specific immune response and immune modulation ex vivo. The described model allows high throughput screening of immunemodulatory therapies on anti-tumor $\mathrm{T}$ cell functions.

Methods Primary patient tumor fragments simultaneously implanted in NOG mice to establish PDX and in parallel were placed in culture to expand primary TIL ex vivo. PDXO established from resected xenograft tumors were characterized for cancer marker expression and tumor histology. Expanded TIL were characterized by flow cytometry. A 3D autologous co-culture using fluorescently labeled PDXO and matching labeled patient TIL were incubated for four days with and without anti-PD1 treatment. High content imaging was used to measure $\mathrm{T}$ cell infiltration and tumor-specific cytotoxicity. Flow cytometry analysis was used to further evaluate $\mathrm{T}$ cell function with and without immunomodulatory therapy.

Results PDXO were established and characterized to mimic in vivo tumor biology and histology. TIL were successfully expanded and characterized to express memory, inhibitory, activation, and regulatory $\mathrm{T}$ cell markers. In cocultures, TIL infiltration in PDXO was observed by high content imaging and confirmed using immunofluorescence detection of CD8+ $\mathrm{T}$ cells within the PDXO. Immune infiltration in the PDXO and resultant tumor cell killing were quantified in response to immunotherapeutic intervention. Treatment with immune-modulatory therapeutics impacted $\mathrm{T}$ cell infiltration and tumor cytotoxicity. Flow cytometry analysis further elucidated the impact of co-culture and drug treatment on $\mathrm{T}$ cell phenotype and functional activity.

Conclusions This autologous, patient-derived, 3D ex vivo platform provides an improved model for evaluating therapeutic efficacy and pharmacodynamics of therapeutic drugs, while simultaneously providing a window into $\mathrm{T}$ cell-tumor cell interactions constrained within the existent patient $\mathrm{T}$ cell repertoire and autologous MHC restriction.

http://dx.doi.org/10.1136/jitc-2021-SITC2021.004 